\title{
Practical Considerations for Ultrashort Electron Pulse Characterization in Ultrafast Transmission Electron Microscopy
}

\author{
Dayne Plemmons and David J. Flannigan
}

Department of Chemical Engineering and Materials Science, University of Minnesota, Minneapolis, MN, 55455, USA

In ultrafast transmission electron microscopy (UTEM), extension of the static analytical capabilities of transmission electron microscopy (TEM) to the ultrafast temporal domain relevant for many atomicscale processes allows for direct visualization of non-equilibrium structural phenomena [1]. Analogous to pump-probe spectroscopic techniques, atomic-scale spatiotemporal resolution is accomplished by operating a properly-modified TEM in stroboscopic mode. In this mode, an ultrafast laser pulse is divided into a pump beam used to excite the specimen in situ and a probe beam used to produce an ultrashort electron packet in the gun region; precise relative timing of the arrival of the laser pulse and electron packet at the specimen enables acquisition of information at specific time points during the dynamic response of the specimen. In this approach, temporal resolution is ultimately limited by the finite duration of the pump laser pulse and probe electron packet. Still, for dynamics occurring on a time scale comparable to the instrument response function, determination of temporal properties of the pulses in UTEM is critical for isolating the intrinsic dynamics.

All-optical methods for determining durations of femtosecond laser pulses are well-established and rely on interaction of two overlapping pulses within a non-linear crystal to produce a second-harmonic signal that varies with time. Extension of this technique to the characterization of ultrashort electron pulses relies on coupling of the photoelectrons with an intense laser field; however, energy-momentum conservation mandates that absorption or stimulated emission of a photon by an electron can only occur in the presence of a third body [2]. In practice, this requirement can be fulfilled both for electrons ionized from bound atomic states via optical excitation of a metal surface [3] and freely-propagating electrons passing through an evanescent near-field of a nanostructure [4]. The latter case is particularly intriguing for the characterization of accelerated electron pulses used in ultrafast electron imaging and diffraction, as the specimen can act as the third-body facilitating free-free transitions. Thus, pulse properties extracted are those precisely at the specimen location. This has recently been observed and described, along with the energy-time correlation of the electron packets, for a $200 \mathrm{kV}$ UTEM [5].

Here, we will discuss considerations for isolating artifacts of the highly non-linear near-field interactions from the true pulse characteristics. Using theory developed to describe these interactions [6], we will discuss how temporal cross-sections of peaks in the electron energy-loss spectra corresponding to higher-order transitions are expected to exhibit the true temporal behavior of the electron pulses. In general, the exceedingly small portion of the pump laser pulse capable of initiating these high-order transitions results in observation of temporal widths converging to the electron packet duration. Additionally, population of transition states occurring for an electron beam focused on the edge of a nanostructure suggest that the energy distribution may result in well-defined chromatic aberrations in images arising from the velocity dependence of magnetic lens focusing. As such, we will discuss the prospect for detecting such phenomena and its potential as a means of determining the instrument response without the need for a spectrometer. Appropriate interpretation of observed spectroscopic and 
image features should in principle enable systematic temporal and spatial deconvolution allowing for a more accurate depiction of the intrinsic ultrafast dynamics.

\section{References:}

[1] D. J. Flannigan and A. H. Zewail, Acc. Chem. Res. 45 (2012), 1828.

[2] A. Weingartshofer, J. K. Holmes, G. Caudle, E. M. Clarke and H. Krüger, Phys. Rev. Lett. 39

(1977), 269.

[3] G. Saathoff, L. Miaja-Avila, M. Aeschlimann, M. M. Murnane and H. C. Kapteyn, Phys. Rev. A. 77 (2008), 022903.

[4] B. Barwick, D. J. Flannigan and A. H. Zewail, Nature 462 (2009), 902.

[5] S. T. Park, O.-H. Kwon and A. H. Zewail, New J. Phys. 14 (2012), 053046

[6] S. T. Park, M. Lin and A. H. Zewail, New J. Phys. 12 (2010), 123028.

[7] This work is partially supported by $3 \mathrm{M}$ through a Non-tenured Faculty Award. Acknowledgment is made to the Donors of the American Chemical Society Petroleum Research Fund for partial support of this research.

a

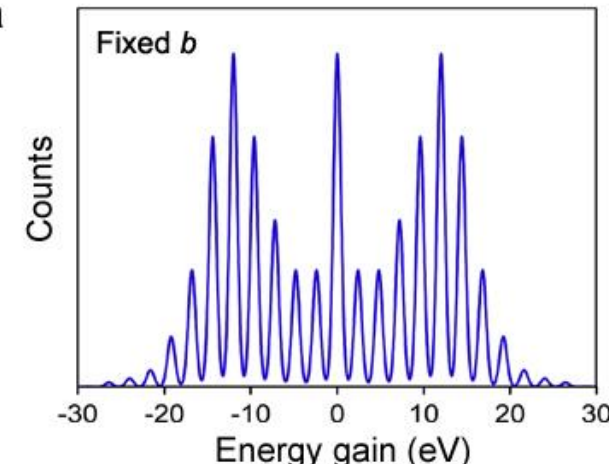

PINEM aberration

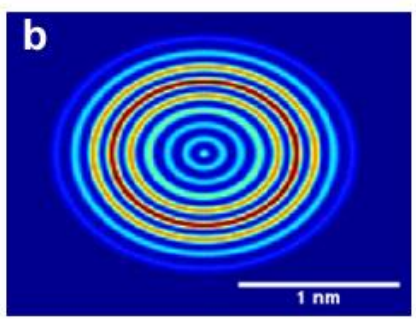

\section{Resulting point-spread function}

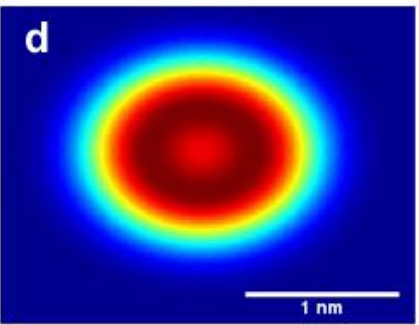

Figure 1. (a) EELS data corresponding to occupation of virtual transition states showing a coherent electron energy distribution. (b) Real-space annular point-spread function due to chromatic aberration arising from population of the quantized virtual states, dubbed a PINEM aberration, where PINEM stands for photon-induced near-field electron microscopy. (c) Point-spread function due to typical spherical aberration present in the objective lens. Convolution of the PINEM and spherical aberration results in the overall point-spread function shown in (d). (e) Time-dependent variation of Fourier intensity for a discrete frequency arising from the convoluted aberrations in the real-space images. The overall response ultimately arises from a convolution of the Gaussian electron and photon packets. 